\title{
Magnetism in Pd: Magnetoconductance and transport spectroscopy of atomic contacts
}

\author{
F. Strigl, ${ }^{\prime}$ M. Keller,' D. Weber, ${ }^{\prime}$ T. Pietsch, ${ }^{2}$ and E. Scheer ${ }^{1, *}$ \\ 'Universität Konstanz, Fachbereich Physik, D-78457 Konstanz, Germany' \\ ${ }^{2}$ Universität Konstanz, Zukunftskolleg und Fachbereich Physik, D-78457 Konstanz, Germany \\ (Received 7 July 2016; revised manuscript received 28 September 2016; published 24 October 2016)
}

\begin{abstract}
Since the rapid technological progress demands for ever smaller storage units, the emergence of stable magnetic order in nanomaterials down to the single-atom regime has attracted huge scientific attention to date. Electronic transport spectroscopy has been proven to be a versatile tool for the investigation of electronic, magnetic, and mechanical properties of atomic contacts. Here we report a comprehensive experimental study of the magnetoconductance and electronic properties of Pd atomic contacts at low temperature. The analysis of electronic transport $(d I / d V)$ spectra and the magnetoconductance curves yields a diverse behavior of Pd single-atom contacts, which is attributed to different contact configurations. The magnetoconductance shows a nonmonotonous but mostly continuous behavior, comparable to those found in atomic contacts of band ferromagnets. In the $d I / d V$ spectra, frequently, a pronounced zero-bias anomaly (ZBA) as well as an aperiodic and nonsymmetric fluctuation pattern are observed. While the ZBA can be interpreted as a sign of the Kondo effect, suggesting the presence of magnetic impurity, the fluctuations are evaluated in the framework of conductance fluctuations in relation to the magnetoconductance traces and to previous findings in Au atomic contacts. This thorough analysis reveals that the magnetoconductance and transport spectrum of $\mathrm{Au}$ atomic contacts can completely be accounted for by conductance fluctuations, while in Pd contacts the presence of local magnetic order is required.
\end{abstract}

DOI: 10.1103/PhysRevB.94.144431

\section{INTRODUCTION}

Atomic contacts have been proven to be of fundamental importance for the advancement and understanding of mesoscale physics. Within the past three decades, atomic contacts of many different materials have been fabricated via scanning tunneling microscopy (STM), mechanically controllable break junctions (MCBJ), or electromigration techniques [1] and were analyzed in terms of their electronic and magnetoconductance properties. With these experiments in conjunction with extensive theoretical modeling, a great variety of transport phenomena were identified and understood, including vibronic and magnonic excitations as well as zero-bias anomalies and conductance fluctuations [2-5]. However, the analysis of experimental data obtained from atomic contacts remains challenging due to the limited knowledge of the geometric contact configuration. Therefore a statistical and holistic investigation of electronic, magnetic, and mechanical properties of the contact is necessary for an adequate description of the experimental evidence. Here we present a comprehensive study of the magnetoconductance and spectroscopic characteristics of $\mathrm{Pd}$ atomic contacts.

Besides its technical importance, $\mathrm{Pd}$ is of particular interest due to its electronic structure. Similar to $\mathrm{Pt}$, it is a strong paramagnet close to the Stoner transition to ferromagnetism, meaning it is nonmagnetic in bulk but predicted to develop magnetic order in reduced dimensions. There are many theoretical and experimental investigations on small clusters which support this scenario [6-12]. However, atomic contacts and chains [13-15] constitute a special geometry in reduced dimensions.

An additional challenge in the calculation is taking into account the coupling of the contact to the constricted electrodes, the atomic configuration of which deviates from

\footnotetext{
"elke.scheer@uni-konstanz.de
}

the bulk crystal structure [16]. Although a multitude of theoretical investigations exist which address the electron transport in such contacts and chains [17,18], experimental reports are scarce. So far a single experimental study reports on the magnetoconductance of Pd nanoconstrictions [19] with diameters of 5-20 nm. Additionally, one recent report exists on the electronic transport characteristics of $\mathrm{Pd}$ tunnel junctions [20], but none in the limit of atomic contacts. In Ref. [19] large magnetoconductance effects at field scales of only a few tens of millitesla have been reported in Pd nanojunctions and were interpreted as a sign of magnetic order. Similarly, our study on the magnetoconductance of Pt atomic contacts evidenced the existence of magnetic order at the atomic scale [21]. In contrast to the findings in nanometer-sized Pd, the effects in Pt vanished for contacts larger than a few nanometers. Such discrepancy between Pd and Pt would not be expected from theoretical predictions of $a b$ initio transport calculations [12,22-24] and experimental evidence derived from small metal clusters $[7,11,25]$.

Another recent study focused on the analysis of the magnetic properties of atom-sized contacts made of $3 d$ magnets, where a ZBA in the $d I / d V$ is present and was attributed to the Kondo effect [26,27]. In Ref. [27] characteristic differences for the Kondo parameters of $\mathrm{Fe}, \mathrm{Co}$, and $\mathrm{Ni}$ contacts were elaborated, signifying their distinct electronic structure and the presence of magnetism in these systems. Herein, we extend our earlier analysis of the magnetoconductance in $\mathrm{Pt}$ atomic chains to the comprehensive analysis of spectroscopic features and magnetotransport of $\mathrm{Pd}$ atomic contacts.

\section{EXPERIMENTAL METHODS}

\section{A. Sample fabrication}

Pd atomic contacts were fabricated via the MCBJ technique. The samples consist of two metallic leads which are connected 
(a)

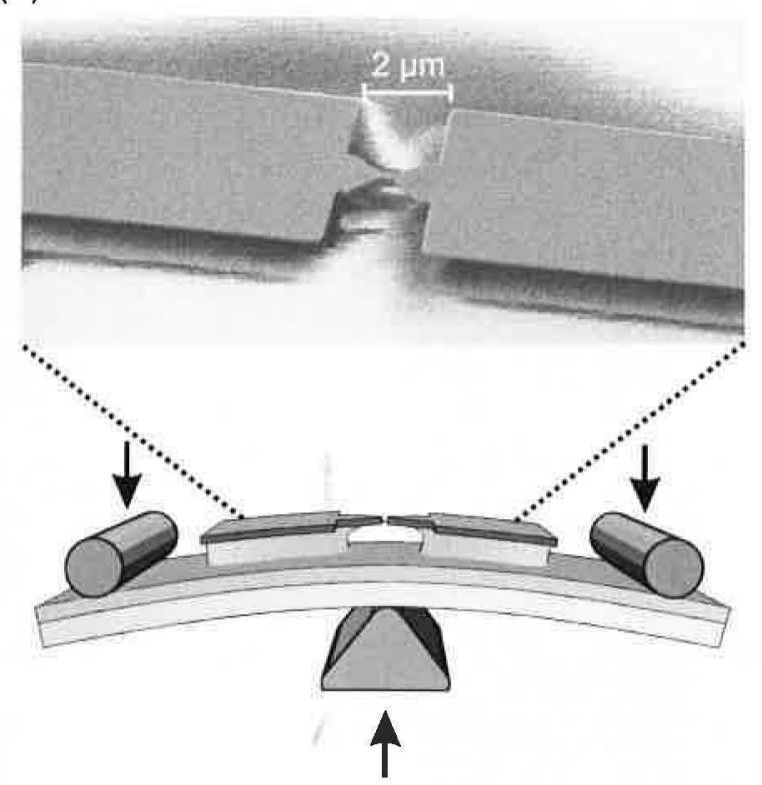

(b)

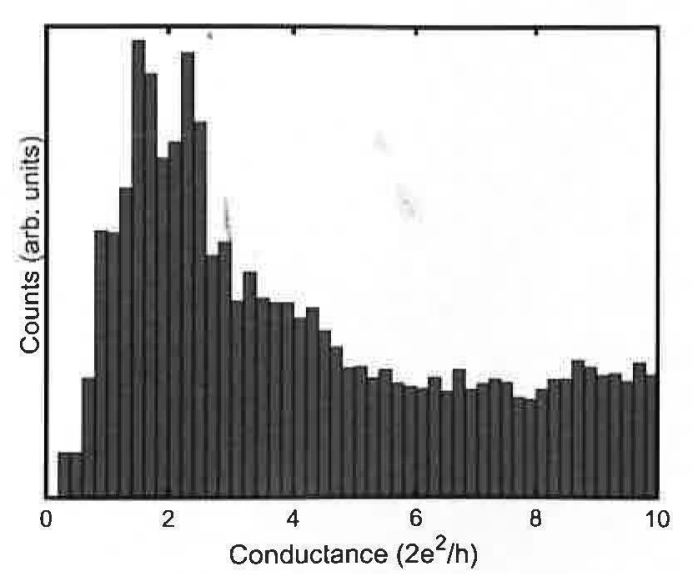

FIG. 1. (a) Scheme of a mechanically controllable break junction (MCBJ) setup. Freestanding atomic contacts and chains are created from a thin constriction, which connects two broad leads, when the sample is bent in a three-point breaking mechanism. The movements of the pushing rod and countersupport are indicated by the arrows. The upper panel shows a SEM micrograph of a Pd sample. (b) Conductance histogram calculated from 349 opening traces of an electromigrated Pd-MCBJ. A broad peak in the conductance histogram between 1.0 and $2.5 G_{0}$ indicates the preferred conductance of a single-atom contact.

by a freestanding bridge of $100 \times 100 \mathrm{~nm}^{2}$ area cross section and are prepared employing the standard fabrication scheme for MCBJs [28]. The sample is then mounted in a mechanical three-point breaking mechanism, which consists of a pushing rod and two countersupports as shown in Fig. 1. Bending of the substrate translates into strain in the constriction, which eventually leads to the controlled breaking of the contact in the constriction. The combination of breaking mechanism and sample design enables us to adjust the electrode separation with subpicometer precision and keep the sample mechanically unperturbed for time scales up to several weeks.

\section{B. Electronic transport measurements}

Electronic measurements are performed using a quasi-fourpoint method in cryogenic vacuum at low temperatures. A constant voltage is supplied to the sample and a high-Ohmic $(100 \mathrm{k} \Omega$ ) reference resistance that is connected in series to the sample and defines the current. The voltages as well as the differential voltages over the sample and reference resistor are measured simultaneously with multimeters and by means of the lock-in technique. After cooldown in a cryogenic vacuum to $4.2 \mathrm{~K}$ we perform a soft electromigration process to reduce crystal defects and remove contaminations from the fabrication process in the constriction [21]. Subsequently, a conductance histogram is recorded by repeatedly opening and closing the junction at low applied de voltage of $\sim 1 \mathrm{mV}$ at the sample to determine the preferred conductance values of the Pd atomic contact and verify the purity of the sample. For Pd we find a broad conductance peak at around 1-2.5 $G_{0}$ [see Fig. 1(b)], which is in agreement with previous studies on Pd [29].

Most of the magnetoconductance and current-voltage traces were acquired in the few-atom regime. We carefully opened the contacts and stopped the motor when the desired conductance was reached. Subsequently, measurements of $d I / d V$ transport spectroscopy and magnetoconductance (MC) were performed as described below.

To record MC characteristics a constant current in the range of $10^{-7} \mathrm{~A}$ is fed while applying a variable magnetic field in different directions. Most MC traces were recorded in magnetic fields up to $9 \mathrm{~T}$ perpendicular to the sample plane ( $z$ direction). Additionally, in-plane field scans were performed, where the magnetic field is applied in the sample plane, parallel to the current direction ( $x$ direction), with a maximum amplitude of $3 \mathrm{~T}$. Angle-resolved MC measurements were performed by rotating the magnetic field in the $x z$ plane at a constant field amplitude up to $3 \mathrm{~T}$. Electronic transport spectra are recorded at constant magnetic field by sweeping the voltage supplied to the sample at a constant rate (mostly $1 \mathrm{mV} / \mathrm{s}$ ) in the window of $\pm 35 \mathrm{mV}$ at the atomic contact.

We also investigated the temperature dependence of the current-voltage characteristics of some contacts. An atomic contact was typically established at the base temperature of the cryostat $(1.5-1.8 \mathrm{~K})$, and the temperature was increased stepwise in increments of $1-3 \mathrm{~K}$.

\section{RESULTS AND DISCUSSION}

In the following section, we first discuss the MC characteristics, followed by the analysis of the zero-bias anomalies (ZBAs) in the current-voltage curves of single-atom Pd contacts. Some contacts did not show a pronounced ZBA; these traces are discussed Sec. III C, starting with their temperature dependence, which motivates the subsequent treatment of these characteristics as conductance fluctuations.

\section{A. Magnetoconductance behavior \\ I. Perpendicular field}

Figure 2 shows a collection of typical MC curves found in atomic Pd contacts when a magnetic field is applied in $z$ direction. The MC shows large, nonmonotonous behavior with 


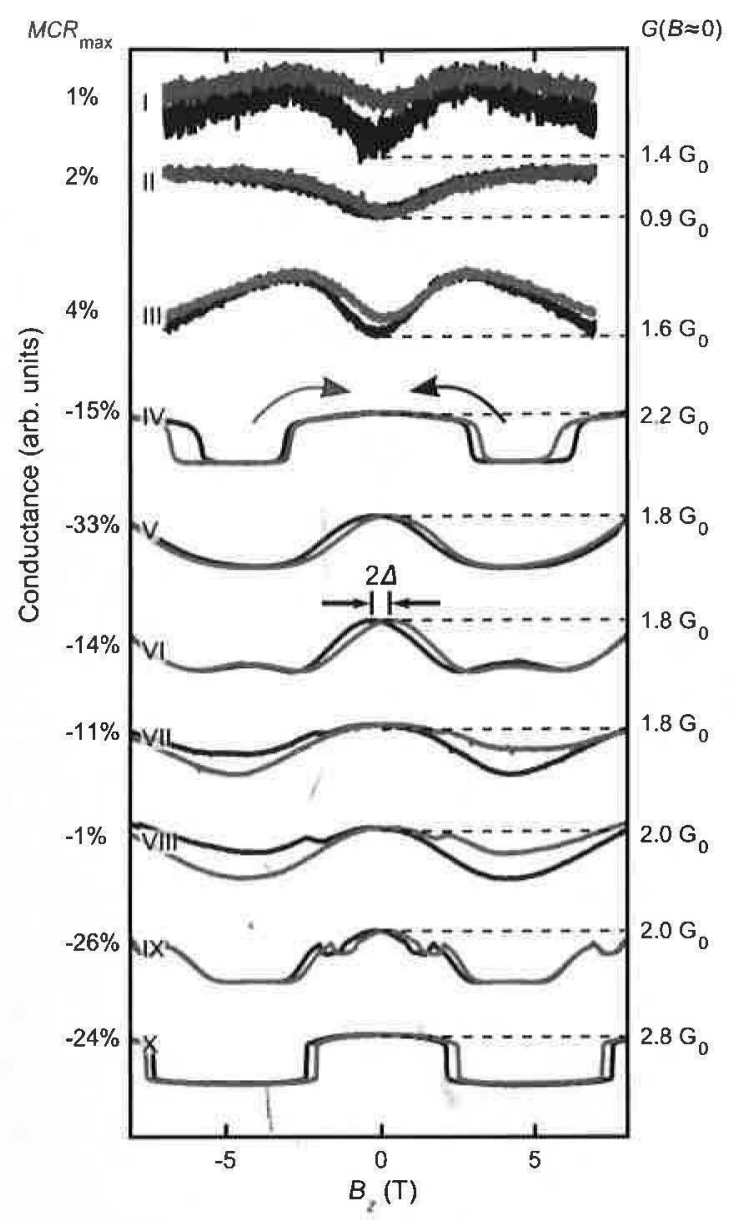

FIG. 2. Selected magnetoconductance curves of atom-sized Pd contacts, where the magnetic field was applied perpendicular to the sample plane ( $z$ direction). The colors indicate the sweep direction of the external magnetic field between the limits of $\pm 8 \mathrm{~T}$ as indicated by the arrows. All curves feature an extremum near zero magnetic field and additional extrema of inverse character symmetrically located at fields of 3-5 T. The labels on the left axis $\left(M C R_{\max }\right)$ indicate the relative differences between these extrema of each curve. The label on the right axis denotes the zero-field conductance of each contact. The hysteresis $2 \Delta$, i.e., the shift of the low-field extrema in the sweep direction of the magnetic field, is indicated exemplarily in curve VI.

varying shape and amplitude. Contacts with a conductance of $\geq 40 G_{0}$ show only a very small linear gradient of the MC.

The most dominant characteristic includes an extremum near $0 \mathrm{~T}$ and two extrema which are located symmetrically at $\left|B_{z}\right| \approx 3-5 \mathrm{~T}$. These finite-field extrema (FFE) are of the opposite type of extreme than the low-field extremum (LFE, $B_{z} \approx 0$ ). In some traces, additional, less pronounced extrema appear superimposed onto the typical behavior. For a quantitative analysis we define the $\mathrm{MC}$ ratio (MCR) as

$$
M C R=\frac{G(B)-G_{\mathrm{ext}}(B \approx 0)}{G_{\mathrm{ext}}(B \approx 0)} \times 100 \%,
$$

where the LFE is denoted by $G_{\text {ext }}(B \approx 0)$. The maximal deviation of the MCR from $G_{\text {ext }}(B \approx 0)$ is denoted as $M C R_{\max }$. We find $M C R_{\max }$ in a range between $-45 \%$ and $+10 \%$, in an approximately 5:1 ratio favoring negative MCR values. However, there is no correlation between the amplitude of the MC, its shape, or zero-field conductance for atomic-scale contacts. The resulting MC characteristics are therefore subject to the exact contact geometry and the local DOS of the contact. This complex and sensitive behavior has attracted the interest of many theorists $[14,15,30,31]$ and has led to different theoretical predictions of the local magnetic order in these contacts. The nonmonotonous and smooth behavior is comparable in size and shape to the characteristics observed in stable atomic contacts made of bulk ferromagnets [32-35]. However, alike contacts made of band ferromagnets, they sometimes display sudden conductance jumps, which were interpreted as signs of rapid geometric contact rearrangements due to magnetostriction [36,37] or other disturbances [38]. This similarity supports the hypothesis of the presence of a local magnetic order in the constriction.

Besides the nonmonotonous behavior, we observe a pronounced hysteresis of the MC as follows: the LFE is shifted in the sweep direction of the magnetic field after application of a high magnetic field ( $\gtrsim 5 \mathrm{~T}$ ), while for lower magnetic fields or newly established contacts it is exactly at $0 \mathrm{~T}$. Such a feature indicates the existence of a coercive field and therefore an existing magnetic order. The hysteresis $\Delta$ determined from the MC measurements lies between 140 and $310 \mathrm{mT}$, which is comparable to earlier superconducting quantum interference device results on small Pd clusters [7,9]. However, the high magnetic anisotropy energies (MAE) calculated for atomic chains $[12,14,22,30]$ and contacts would lead to much higher hysteresis. Hence, the origin of the hysteresis is not attributed to the central contact atom but has to be located in the magnetized electrodes in the direct vicinity to the atomic contact.

\section{Parallel field}

To investigate the anisotropy of the MC, we also applied magnetic fields in the sample plane, parallel to the direction of the current. Most of the recorded traces in $x$ direction show a parabolic behavior without hysteresis. The $\left|M C R_{\max }\right|$ in $x$ direction is mostly between 0 and $4 \%$, with one exceptional trace revealing $M C R_{\max } \approx 15 \%$. Occasionally irregular traces appear, as shown in Fig. 3, which may be caused by very unique contact configurations and are not discussed any further. The lacking hysteresis in most curves in comparison to the $z$ sweeps may be a consequence of the limited field range $(<3 \mathrm{~T})$ in this direction, which is substantially lower than the high-field extremum observed in most $\mathrm{MC}$ traces in $z$ direction. It will be shown later that the position of the FFE is associated to the alignment of the electrode's magnetization, which also determines the hysteresis. One important observation is that the $\mathrm{MC}$ traces of the same contact recorded in $x$ and $z$ direction are always of opposite sign, indicating the anisotropic contribution of conductance channels as described for $\mathrm{Ni}$ atomic contacts [39]. Markedly, the MCR in moderate fields up to $3 \mathrm{~T}$ has an approximately parabolic shape, irrespective of the field direction (parallel $x$ or perpendicular $z$ ), but with different vertical scale factors. In particular, we find that negative $\mathrm{MC}$ always leads to larger $M C R_{\max }$ values than the positive ones. This observation is independent of the direction of the applied magnetic field but seems to be an intrinsic behavior of these contacts. 


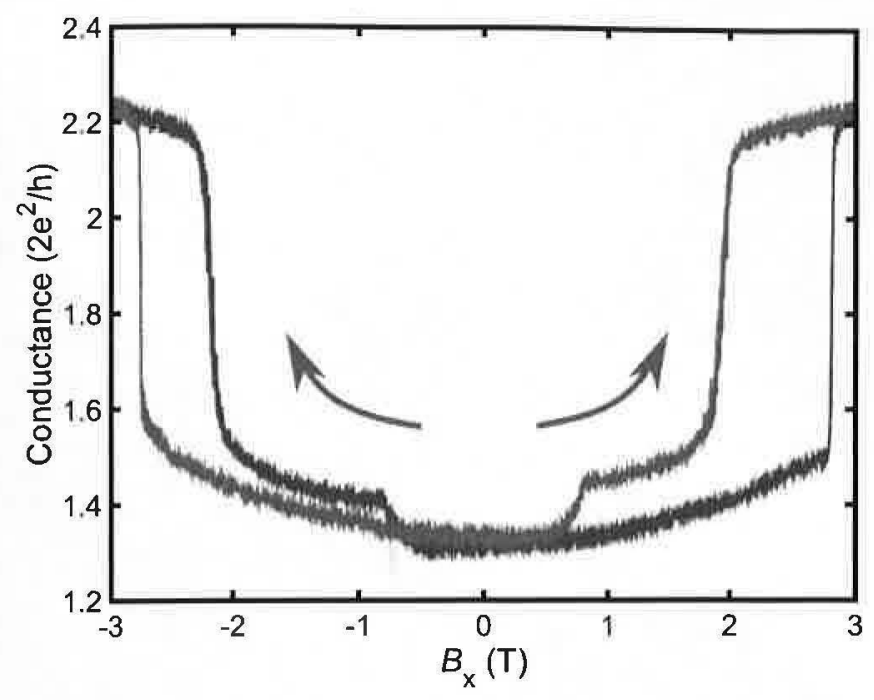

FIG. 3. An irregular MC trace of an atom-sized Pd contact recorded in magnetic field applied in $x$ direction. A large conductance change $\left(M C R_{\max }=70 \%\right)$ occurs in the range of $2-2.8 \mathrm{~T}$ depending on the sweep direction, indicated by the arrows. In contrast, the majority of Pd contacts show parabolic magnetoconductance curves when the field is applied in $x$ direction with moderate $\left|M C R_{\max }\right|$ between $1 \%-15 \%$.

\section{Angular dependence}

By performing a vector sweep in the $x z$ plane, we record the anisotropic magnetoconductance (AMC)

$$
A M C=\frac{G(\theta=0)-G(\theta)}{G(\theta=0)} \times 100 \%,
$$

where $\theta$ denotes the angle between the direction of the current and the applied magnetic field. The amplitude of this effect is in the range of $10 \%$. Such a high amplitude is not expected for a paramagnet but has been found in atomic contacts of bulk ferromagnets [39-41]. The $A M C$ found here (Fig. 4) does not follow the trigonometric $\sin ^{2} \theta$ shape expected for bulk ferromagnets; the curvature is stronger around the minima at $\theta \sim 0$ and $-\pi$ but weaker around the maxima at $\theta \sim-\pi / 2$ than a $\sin ^{2} \theta$. This deviation from the $\sin ^{2} \theta$ shape can also be deduced from parabolic $x$ - and $z$-magneto sweeps, discussed in the previous sections, which open with different scale factors depending on the sign of the magnetoconductance. Moreover, compared to the typical $\sin ^{2} \theta$ shape, we observe a phase shift of the $A M C$. Similar effects have been found in atomic-size $\mathrm{Ni}$ contacts and were shown to originate from the exact atomic transport channel composition [39].

\section{Microscopic contact model}

All observations made here so far are comparable with earlier results on atomic Pt contacts [21] but in stark contrast to the features reported for nanoscale Pd contacts [19] where the effects are monotonous, much larger, and occur at larger contact sizes and lower magnetic fields. These discrepancies may be due to the different sample design, since the samples investigated in Ref. [19] are suspended over a length of at least $100 \mu \mathrm{m}$ (cf. Ref. [1]) and are therefore prone to magnetostric-

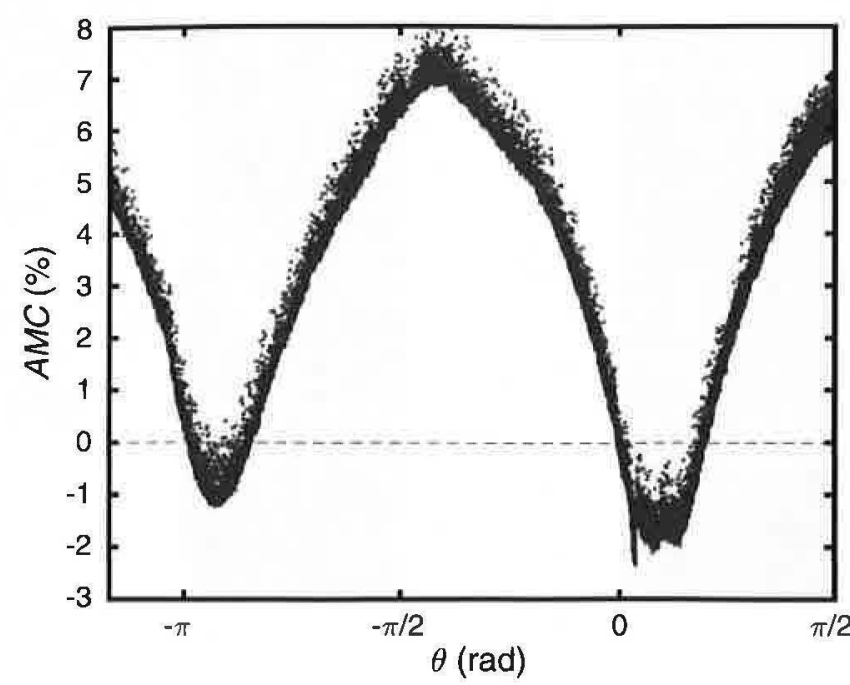

FIG. 4. Typical anisotropic magnetoconductance $(A M C)$ of an atom-sized Pd contact with zero-field conductance of $2.4 G_{0}$. The field is rotated in the $x z$ plane at a constant field amplitude of $3 \mathrm{~T}$; the field angle $H=0$ corresponds to a magnetic field in $x$ direction. The AMC amplitude is approximately $10 \%$, similar to that in atomic contacts of ferromagnets.

tion, while this can be excluded in our experiments, since large contacts $\left(G>40 G_{0}\right)$ do not show any MC effect [36].

In $\mathrm{Pt}$ atomic contacts and chains, five observations indicated the emergence of magnetic order. These were (i) the size and (ii) shape of the MC and $\mathrm{AMC}$, which were comparable to those found in atomic contacts of band ferromagnets. Thirdly (iii), both MC and AMC strongly depended on the atomic contact configuration and changed upon stretching the contact by a fraction of an atomic diameter. The fourth indication (iv) was the observation of a hysteresis which strongly depended on the exact contact geometry. Lastly (v), the in-plane MC was strongly dependent on the history of the contact with respect to fields applied in $z$ direction.

Although Pd and Pt share those general characteristics, there are distinct differences, which we will discuss in the following:

(1) The fraction of negative to positive $M C R_{\max }$ in $z$ direction is significantly higher for $\mathrm{Pd}(5: 1)$ than for $\mathrm{Pt}(2: 1)$.

(2) In $\mathrm{Pd}$ the hysteresis $\Delta$ reveals a smaller spread $(140-310 \mathrm{mT})$ than in $\mathrm{Pt}(0-800 \mathrm{mT})$.

(3) $\mathrm{Pd}$, on average, shows larger values of $\left|M C R_{\max }\right|$ in the $z$ field but smaller values when measured in $x$ direction.

(4) In Pd the outer extrema are located at lower magnetic fields (3-5 T) than in Pt (4-7 T).

The microscopic origin of these differences may be understood considering the atomic and electronic configuration of the junctions and their effect on the MC behavior. In a minimal model, similar to the one reported for $\mathrm{Pt}$ [21], the junction consists of three distinct parts, i.e., the central atom, the apex atoms, and the tips of the electrodes (see also Fig. 5). All atoms in the contact area contribute to the magnetic order, while their contributions are characterized by different MAEs and magnetic moments. Depending on the exact configuration, a large variety of possible, stable magnetic states can occur, including noncollinear arrangements. Each of the moments 
(a)

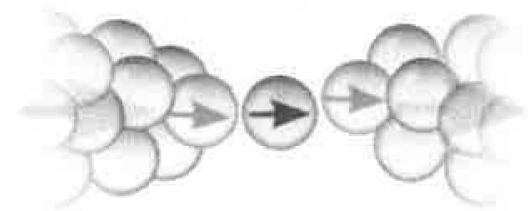

(b)

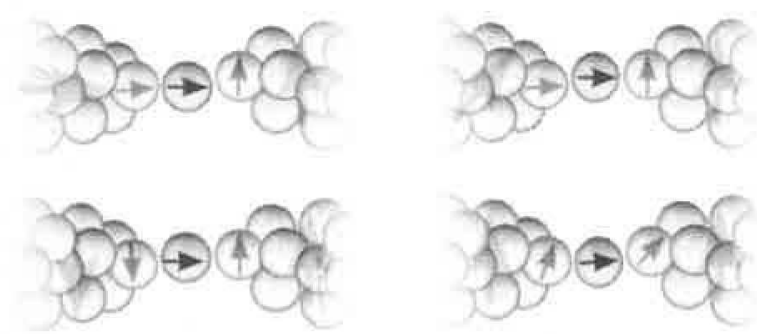

FIG. 5. The magnetic state in an atomic-size contact is defined by contributions of different parts: the electrodes (blue), the apex atoms (magenta), and the central atom (cyan). Each of these parts is characterized by a different magnetic moment and magnetic anisotropy energy, which depend on the electronic and geometric environment. The direction of local magnetic moments is indicated by the arrows. Panel (a) shows a ferromagnetic (collinear) alignment of the magnetic moments. This is the most likely ground-state configuration, which results in a negative $M C R_{\max }$ as observed in the majority of Pd contacts, e.g., curves IV-X in Fig. 2. (b) The interpretation of MC curves with a positive $M C R_{\max }>0$ (e.g., curves I-III in Fig. 2) requires a noncollinear ground state; four possible configurations are shown.

in the different parts of the contact responds differently to the application of an external magnetic field, which leads to a relative misalignment of adjacent momenta and causes the observed magnetoconductance.

It is well known that the number of contributing transport channels in atomic contacts is determined by the valence of the element [42] and in contrast to bulk, not only the $s$ electrons contribute to the electron transport [1]. Depending on the contact configuration, the valence orbitals hybridize differently to form the transport channels $[16,43,44]$, which are directly affected by an external magnetic field and contribute to the observed MC.

(a) Variability of observed MC and hysteresis. One of the main differences between $\mathrm{Pd}$ and $\mathrm{Pt}$ stems from the fact that Pd does only form single-atom or dimeric contacts, while $\mathrm{Pt}$ does form chains $[45,46]$. Chain formation in $\mathrm{Pt}$ is also accompanied by surface reconstructions [45]. The lack of surface reconstruction and chain formation in $\mathrm{Pd}$ leads to a smaller number of stable contact configurations than for Pt. The multitude of atomic configurations is directly reflected in the variance of the local density of states (LDOS). Another difference is the lower spin-orbit coupling (SOC) in $\mathrm{Pd}$ compared to $\mathrm{Pt}[30]$. The intricate interplay of different contact configurations, SOC, and LDOS determines the MAEs of the different parts in the constriction [12,24,47] and the magnetotransport properties [12,48-50]. Hence, in Pd one expects a narrower distribution of hysteresis values as well as a lower variability of the $\mathrm{MC}$ behavior compared to $\mathrm{Pt}$.

(b) Amplitude of MCR. Calculations for atomic chains predict a higher magnetic moment per atom for $\mathrm{Pd}$ than for $\mathrm{Pt}$
$[17,18,51,52]$. This implies a larger spontaneous spin-splitting in the LDOS of Pd, particularly in the $d$ orbitals. When a magnetic field is applied, the overlap of wave functions between adjacent atoms in the contact changes and one expects a larger MCR in Pd than in Pt.

(c) Shape of MC traces and position of outer extremes. The larger magnetic moment of Pd implies a higher Zeeman energy, which relates to a stronger torsional force on the localized magnetic momenta, if present, in Pd atomic contacts. This would explain the position of the FFEs at lower fields in Pd, because their location reflects the complete misalignment of localized momenta in the contact, i.e., the relative orientation of the magnetic momenta of apex atoms, electrodes, and the central atom (see Fig. 5).

Another argument for the lower position of the FFEs in Pd takes into account SOC, which leads to an enhanced stability of the magnetic order in Pt $[52,53]$ and therefore to a higher external field required to excite the magnetic ground state. Moreover, the SOC induces spin mixing and promotes the formation of a noncollinear ground state, especially in $\mathrm{Pt}$ chains with stretched bonds, which translates into a positive MC. In Pd, on the other hand, a collinear ground state is more favorable due to the absence of chains and the lower SOC. This explains the large fraction of negative MCRs in Pd.

In conclusion, the striking similarities between the $\mathrm{MC}$ behavior of $\mathrm{Pd}$ and $\mathrm{Pt}$ as well as the band ferromagnets witness the existence of local magnetic order in atom-size Pd contacts. The difference between Pd and earlier reports on $P t$ are understood considering the atomic structure and energy landscape of the contacts.

\section{B. Electronic transport spectroscopy: Kondo resonances}

Electron transport spectroscopy is a highly versatile tool for the investigation of the electronic properties of mesoscopic systems. Here we analyze more than 900 different atomic Pd contacts. Two of the most intriguing $d I / d V$ spectra are presented in Fig. 6.

Generally, the $d I / d V$ transport spectra of $\mathrm{Pd}$ atomic contacts exhibit at least one of the following characteristics: (i) a prominent ZBA and (ii) a nonperiodic oscillatory behavior that vanishes at bias voltages above $\sim 12 \mathrm{mV}$. We employ two different analysis methods to separate and quantify both phenomena. The ZBA in the transport spectra is analyzed in the framework of the Kondo effect and is discussed in this section. Then, in Sec. IIIC we discuss the nature of the oscillatory behavior in the framework of conductance fluctuations.

The Kondo effect is a well-known many-body phenomenon, where localized magnetic moments in a system below the Kondo temperature $T_{\mathrm{K}}$ are shielded by the conduction electrons, which produces a characteristic Kondo resonance in the transport spectra [54,55]. Recently Calvo et al. reported a detailed analysis of Kondo effect in atomic contacts of $3 d$-band ferromagnets [26,27], which showed that the localized $d$ orbital of the central atom can be treated as a quantum dot weakly coupled to the electrodes. The signature of the Kondo effect strongly depends on the nature of the magnetic impurity and the coupling to its environment [26,56-58], as well as the interaction of the $s$ and $d$ orbitals of the central atom [26]. 
(a)

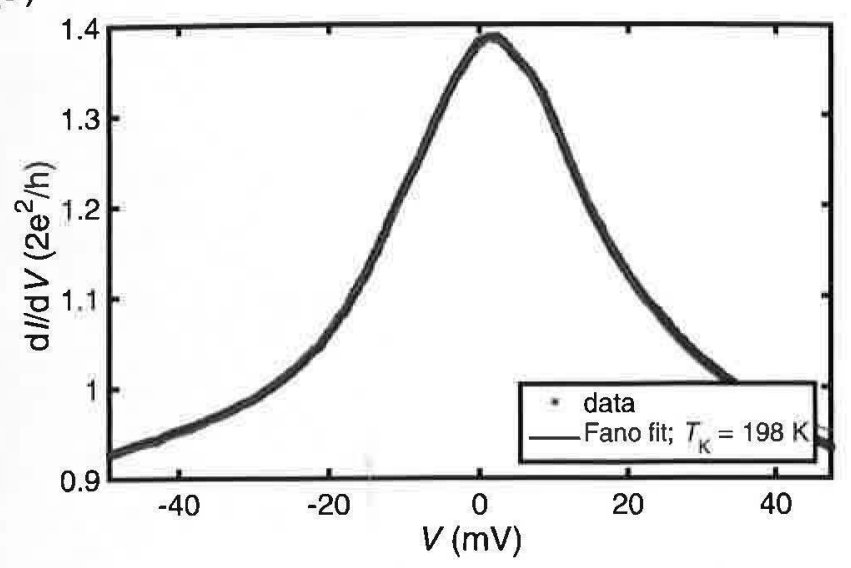

(b)

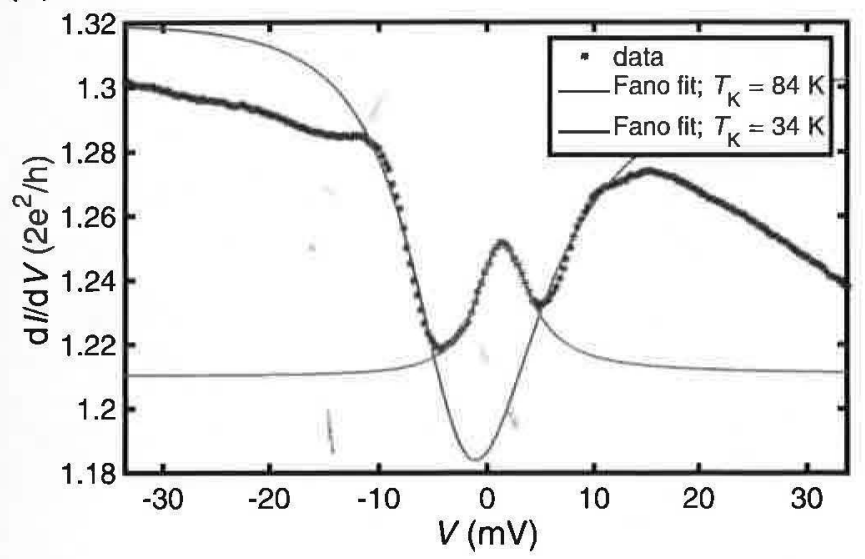

FIG. 6. Two examples of $d I / d V$ spectra of atom-sized Pd contacts showing a zero-bias anomaly (ZBA). (a) ZBA described by a single Kondo resonance fitted with a Fano function (red) and $T_{K}=198 \mathrm{~K}$. (b) Example in which the spectrum is fitted by two independent Fano functions, one for a low-voltage ZBA (pink) and a more pronounced feature vanishing at higher voltages (red).

Following Ref. [27], we analyze the Kondo resonance by fitting a Fano function of the form

$$
\frac{d I}{d V}=g_{0}+\frac{A}{1+q^{2}} \frac{(q+\epsilon)^{2}}{1+\epsilon^{2}},
$$

where $g_{0}$ is the linear part of the conductance, $A$ is the amplitude, $q$ is the Fano parameter which defines the shape of the resonance, and $\epsilon=\left(e V-\epsilon_{\mathrm{s}}\right) / k_{\mathrm{B}} T_{\mathrm{K}}$, with $\epsilon_{\mathrm{s}}$ a possible shift of the center of the resonance with respect to the Fermi energy, $\mathrm{eV}$ is the electron energy, and $k_{\mathrm{B}}$ is the Boltzmann constant.

Approximately $43 \%$ of all transport spectra reveal a distinct ZBA that was analyzed using this model. In some contacts, two resonances at different energy scales appear, such as shown in Fig. 7(b). However, often a model of two superimposed Kondo resonances did not converge. Therefore, in these cases we analyze only the resonance featuring the lower $T_{\mathrm{K}}$ and disregard the other. (a)

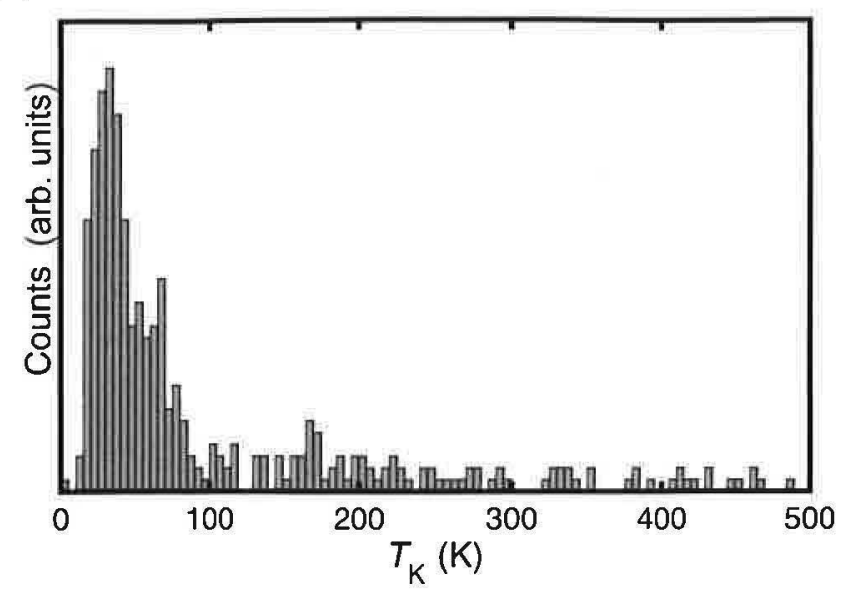

(b)

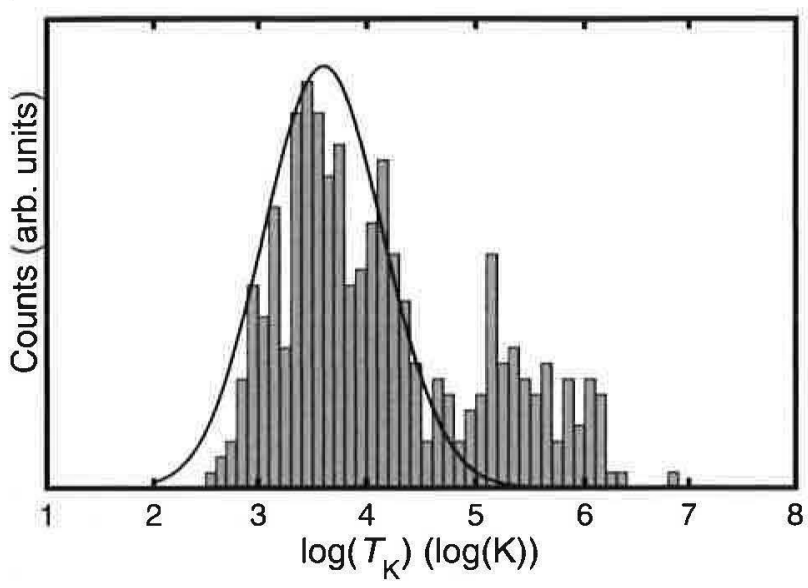

FIG. 7. Histogram of Kondo temperatures $T_{\mathrm{K}}$ extracted from 394 $d I / d V$ spectra of different contacts. (a) The Kondo temperatures are mainly distributed between 20 and $100 \mathrm{~K}$, with a maximum at $\sim 30 \mathrm{~K}$, but there are also Kondo temperatures up to almost $500 \mathrm{~K}$. (b) The same data as shown in (a) in log-normal presentation. As a guide to the eye a log-normal distribution (blue solid line) matching the data is shown. In this representation a second maximum appears at higher $T_{\mathrm{K}}$, indicating the presence of another Kondo resonance.

\section{Distribution of Kondo temperatures}

From all fits, we extract the Kondo temperature $T_{\mathrm{K}}$, shown in Fig. 7, and the energy $\epsilon_{\mathrm{s}}$, which is connected to the occupation number $n_{d}$ of the $d$ orbital [59], shown in Fig. 8.

The distribution of Kondo temperatures in Pd atomic contact reveals a relatively broad peak with a maximum around $30 \mathrm{~K}$. Verifying the Kondo nature of the ZBA by splitting the resonance in a magnetic field by the Zeeman effect would require magnetic fields in excess of $20 \mathrm{~T}$.

The Kondo temperature depends exponentially on the energy of the magnetic impurity, which is assumed to originate from localized $d$ electrons in the atomic contact, whose energy depends on the contact geometry [59]. Since the formation of atomic contacts via mechanical deformation is a purely statistical process, a normal distribution of the energy of the $d$ electron is expected [27], which relates to a normal distribution of the logarithmic Kondo temperatures $\left[\log \left(T_{\mathrm{K}}\right)\right]$. 


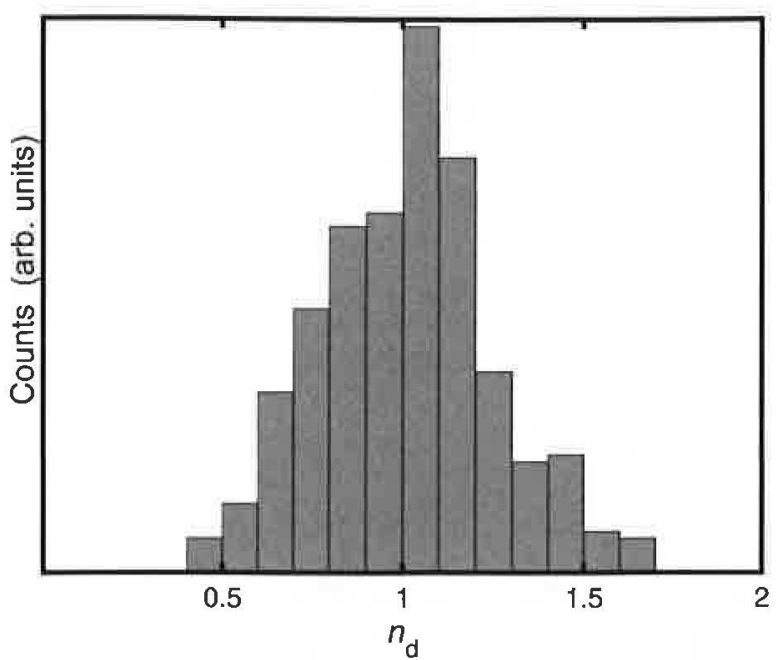

FIG. 8. Distribution of the occupation number of the $d$ orbital, extracted from the fit parameters of ZBAs. The maximum of the distribution is slightly above 1 and the FWHM is $\sim 0.5$ irrespective of the application of the distribution functions (Gauss and Lorentzian).

A histogram of experimentally determined Kondo temperatures $\left[\log \left(T_{\mathrm{K}}\right)\right]$ is shown in Fig. $7(\mathrm{~b})$ together with a log-normal distribution of comparable width and amplitude. The distribution of experimental $T_{\mathrm{K}}$ around $30 \mathrm{~K}$ agrees well with the log-normal expectation, which supports the interpretation that the ZBAs are caused by the Kondo effect. However, a second peak appears in the binary distribution at higher Kondo temperatures, which may be interpreted as the formation of a different class of Kondo impurities at higher energies. This would imply that there are two distinct sets of contact geometries associated to different average Kondo temperatures. In contrast to the distribution of the lower $T_{\mathrm{K}}$, the one at higher temperatures deviates from the expected lognormal behavior, presumably due to the insufficient statistics, because only about $100 d I / d V$ spectra contribute to the high-temperature Kondo peak.

To further elucidate the origin of the binary distribution, we studied the development of $T_{K}$ when a contact is stretched by moving the electrodes a few picometers further apart. The Kondo temperatures extracted from $d I / d V$ spectra recorded within this evolution of contacts always fall into one of the classes of $T_{\mathrm{K}}$. This indicates that there are indeed two distinctly different magnetic configurations possible, which are subject to the exact contact formation.

\section{Kondo occupation number}

The occupation number $n_{\mathrm{d}}$ of the $d$ orbital, which acts as a Kondo impurity, is related to the energy $\epsilon_{\mathrm{s}}$ via [59]

$$
n_{\mathrm{d}}=1-\frac{1}{\pi} \arctan \left(\frac{\epsilon_{\mathrm{s}}}{k_{\mathrm{B}} T_{\mathrm{K}}}\right) .
$$

The occupation numbers extracted from all fitted $d I / d V$ spectra shown in Fig. 8 are distributed between 0.4 and 1.7 with a maximum slightly above 1 . A distribution of occupation numbers in this range is expected in view of the electronic shell structure of $\mathrm{Pd}$. In comparison to the occupation numbers reported for $3 d$ ferromagnets, the width of the distribution

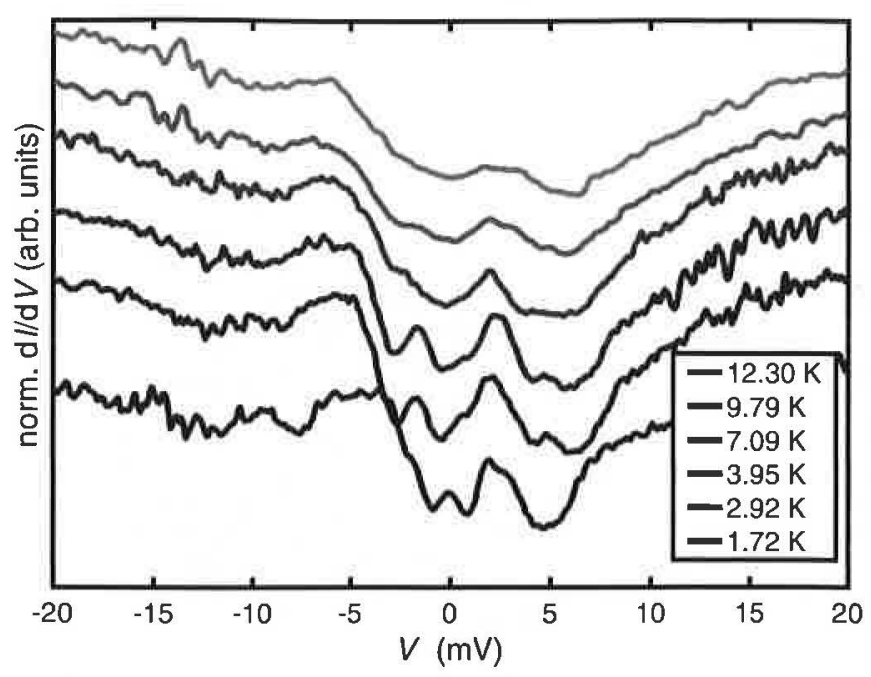

FIG. 9. Typical temperature dependence of transport spectra $(d I / d V)$ without pronounced $\mathrm{ZBA}$, recorded in a single-atom $\mathrm{Pd}$ contact with a conductance of $2.5 G_{0}$. All of the features decrease in amplitude with increasing temperature; most of them eventually vanish at temperatures comparable to the temperature scaling of conductance fluctuations.

(FWHM) for $\mathrm{Pd}(0.5)$ is similar to the one found in $\mathrm{Ni}$ contacts (0.52) [27], which is much smaller than for Fe and Co. This similarity between the distribution of $n_{\mathrm{d}}$ in $\mathrm{Pd}$ and Ni atomic contacts indicates the presence of a spin- $1 / 2$ impurity, which could be expected in view of the isoelectric character of both metals.

\section{Electronic transport spectroscopy: Conductance fluctuations}

In this section, we analyze the oscillatory behavior observed in $d I / d V$ spectra of Pd atomic contacts where no pronounced ZBA appears; a typical example is shown in Fig. 9. The energy scales of these fluctuations are evaluated by probing their temperature dependence.

At temperatures below $4 \mathrm{~K}$, we observe distinct, aperiodic fluctuations for bias voltages up to $\pm 10 \mathrm{mV}$, which decrease in size when the temperature is increased. In most spectra, the fluctuations vanish at temperatures above $\sim 10 \mathrm{~K}$, while sometimes they remain visible even at higher temperatures, such as the peak at $\sim 2.5 \mathrm{mV}$ in Fig. 9. Similar oscillatory behavior in the magnetoconductance and $d I / d V$ characteristics has been reported in different types of mesoscopic conductors $[40,60$ 66]. Their temperature dependence suggests the interpretation as electronic interference effects known as conductance fluctuations (CF) $[62,67,68]$. In atomic contacts interference of partial electronic modes traversing the constriction occurs due to backreflection at the opposing electrode $[5,66]$.

\section{Analysis of CFs in atomic contacts}

CFs are evaluated via autocorrelation analysis $[61,62]$ of the $\mathrm{MC}$ and transport spectra to extract the fluctuation amplitude $\delta G_{\mathrm{rms}}$ as well as the characteristic voltage $V_{\mathrm{C}}$ or coherence field $B_{\mathrm{C}}$, which can be related to the characteristic scattering length. Two boundary cases have to be distinguished: interference paths (i) limited by the phase coherence length of the electronic 
TABLE I. The fluctuation amplitude evaluated using Eq. (5) and extracted from experimental data as a function of both voltage $\left(\delta G_{\mathrm{rns}, \mathrm{V}}\right)$ and magnetic field $\left(\delta G_{\mathrm{rm}, \mathrm{B}}\right)$.

\begin{tabular}{|c|c|c|}
\hline Fluctuation amplitude $\left(e^{2} / h\right)$ & $\mathrm{Au}$ & $\mathrm{Pd}$ \\
\hline$\delta G_{\text {Ims.V.theo }}$ & 0.011 & 0.034 \\
\hline$\delta G_{\mathrm{ms} . \mathrm{V} \cdot \mathrm{exp}}$ & 0.011 & 0.039 \\
\hline$\delta G_{\text {mns.B.exp }}$ & 0.0069 & 0.277 \\
\hline$\delta G_{\text {ms B.exp.sym }}$ & 0.0069 & 0.270 \\
\hline$\delta G_{\text {mms,B.exp, antis }}$ & 0.0008 & 0.050 \\
\hline$\delta(\Delta G)_{\text {me. B.exp }}$ & 0.0014 & 0.064 \\
\hline
\end{tabular}

wave function and (ii) limited by the sample geometry $[69,70]$. We limit the correlation analysis to voltages in the range of $V \leqslant \pm 12 \mathrm{mV}$, since the fluctuation pattern is damped completely by the excitation of phonons [70]. This value is chosen below the bulk phonon modes of Pd (16 meV) [71] in order to account for phonon softening in the atom-sized contact [72,73]. The results of the analysis are compared to those of Au contacts, where conductance fluctuations were intensively studied before [5,21]. Calculations reported in literature $[62,64-66]$ have been performed under very special assumptions and there is no general model for the description of the CFs in atomic contacts. Therefore we use a model for comparison of CFs in $\mathrm{Au}$ and $\mathrm{Pd}$ that was introduced by Holweg et al. [65]. This model successfully describes ballistic but not atomic-size contacts and is based on a combination of the Landauer and Sharvin description of electron transport [74,75]. In this model the voltage-dependent fluctuation amplitude is given by

$$
\delta G_{\text {rms. }} \cong 1.6 \frac{e^{2}}{h} \frac{a}{l_{\mathrm{e}}},
$$

where $e$ is the electron charge, $h$ Planck's constant, $a$ the diameter of the sample, and $l_{\mathrm{e}}$ the electron mean free path. The latter can be estimated by the residual resistance ratio via Matthiessen's rule.

\section{Voltage-dependent fluctuation amplitudes}

A summary of characteristic fluctuation amplitudes of $\mathrm{Au}$ and Pd contacts can be found in Table I. The experimentally determined $\delta G_{\text {rms, } v}$ of $\mathrm{Pd}$ and Au atomic contacts are 0.039 and $0.011 e^{2} / h$, respectively. Using the Holweg model [Eq. (5)], we calculate the theoretically expected values of $\delta G_{\mathrm{ms} . V}$, where the parameter $a$ is given by the atomic diameter of the metal. For Pd, we chose $a=280 \mathrm{pm}$, the empirical atomic diameter, because all traces were recorded in the single-atom regime $\left(0.5-2.5 G_{0}\right)$. For Au we employ an effective diameter of $a_{\text {eff }} \approx 460 \mathrm{pm}$, which is approximately 1.7 times the atomic diameter, because we studied Au contacts in the range of one to three atoms size, with a conductance up to $2.5 G_{0}$.

The elastic mean free paths extracted from the model are $l_{e}(\mathrm{Au}) \sim 65 \mathrm{~nm}$ and $l_{e}(\mathrm{Pd}) \sim 13 \mathrm{~nm}$, respectively. With these parameters, the theoretical values for $\delta G_{\mathrm{rms}, v}$ of $\mathrm{Pd}$ and $\mathrm{Au}$ are 0.034 and $0.011 e^{2} / h$, consistent with the experimental findings. This indicates that the voltage-dependent fluctuation amplitude is determined by the thinnest part of the atomic contact, i.e., the central atom of the contact.

\section{Magnetic-field-dependent fluctuation amplitudes}

The magnetic-field-dependent fluctuation amplitude $\delta G_{\mathrm{ms} . \mathrm{B}}$ is determined from the MC measurements. In $\mathrm{Au}$ atomic contacts, the $\delta G_{\text {rns. B }}$ are similar to $\delta G_{\mathrm{rms}, \mathrm{V}}$, while in $\mathrm{Pd}$ contacts there is a drastic deviation. This observation clearly indicates the presence of a magnetic moment in Pd atomic contacts.

To explain this argument, we first discuss the behavior expected for Au contacts. The experimentally determined $\delta G_{\text {rms. B }}$ in $\mathrm{Au}$ is $0.0069 e^{2} / h$, which is approximately 0.63 times $\delta G_{\mathrm{rms} . \mathrm{V}}$, in good agreement with the theoretical expectation of $\delta G_{\mathrm{rms} . \mathrm{B}}=\delta G_{\mathrm{ms} . \mathrm{v}} / \sqrt{2}$ as a result of lifting the particle-particle and particle-hole symmetries upon the application of a finite magnetic field [62]. Nevertheless, in two-point geometry conductance fluctuations are symmetrical with respect to the applied magnetic field, if time-reversal symmetry is conserved. While this is clearly the case in Au, the MC traces of Pd contacts are not symmetric due to the hysteresis and the sweep-direction-dependent amplitude of the local extrema. This asymmetry indicates the presence of magnetic scattering in the contact, which breaks the timereversal symmetry. In this case, the characteristic properties of CFs may be extracted by evaluating the symmetrized and the antisymmetrized components of the MC. The results of the analysis are shown exemplarily in Fig. 10. The magneticfield-dependent fluctuation amplitudes $\delta G_{\text {rms. B }}$ are higher than $\delta G_{\text {ms. } v}$ for both the symmetrized $\left(0.27 e^{2} / h\right)$ and the antisymmetrized $\left(0.05 e^{2} / h\right)$ components of the $\mathrm{MC}$, which contradicts the theory of conductance fluctuations. However, this result again emphasizes the dominance of magnetic scattering in the contact.

For comparison, the same analysis is performed for the Au contacts. In this case, the antisymmetric component

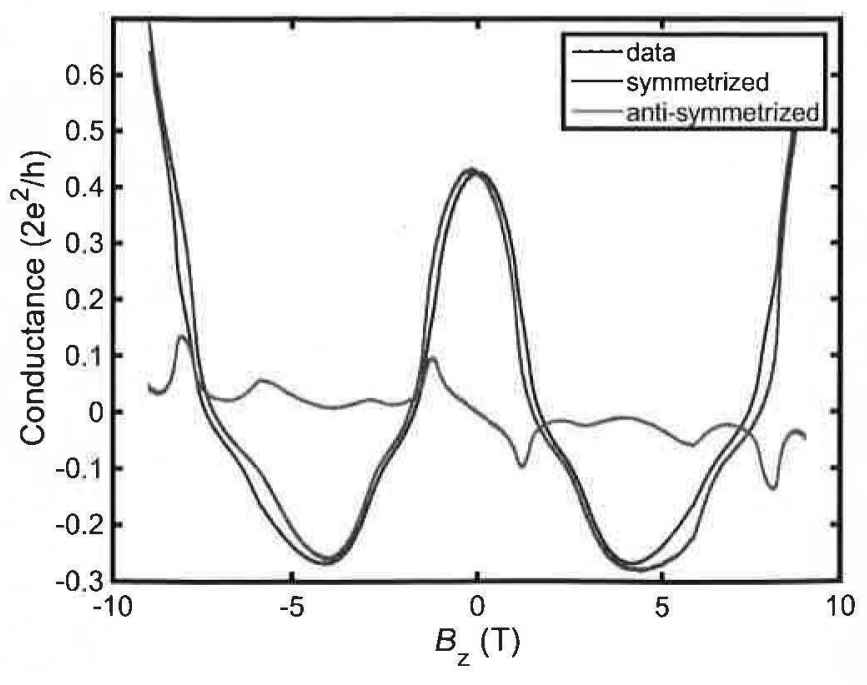

FIG. 10. MC curve of an atom-sized Pd contact with a zero-field conductance of $2.3 G_{0}$ and its symmetric (blue) and antisymmetric (orange) components; the MC data and the symmetric component are shifted for clarity by $-1.9 G_{0}$. The antisymmetric part shows a smaller amplitude $\left(\delta G_{\mathrm{mms}, \mathrm{B}}=0.092 e^{2} / h\right)$ than the symmetric part $\left(\delta G_{1 \mathrm{~ms} \mathrm{~B}}=0.488 e^{2} / h\right)$, which only slightly deviates from the MC $\left(\delta G_{\mathrm{ms \textrm {B }}}=0.496 e^{2} / h\right)$. 


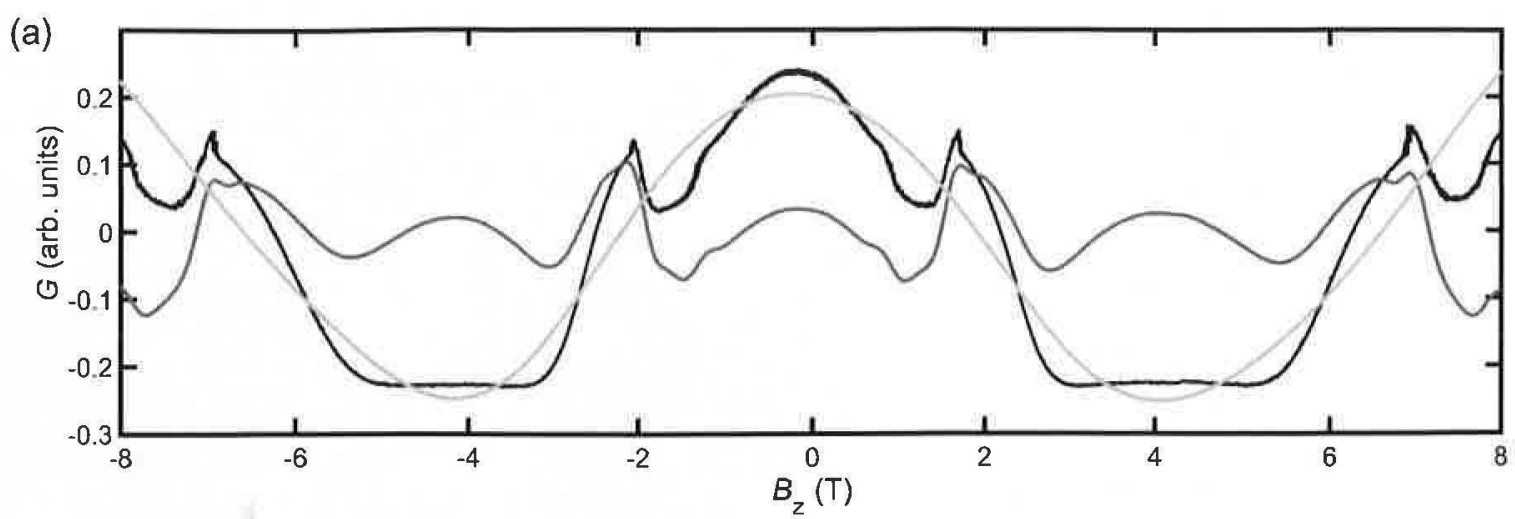

(b)

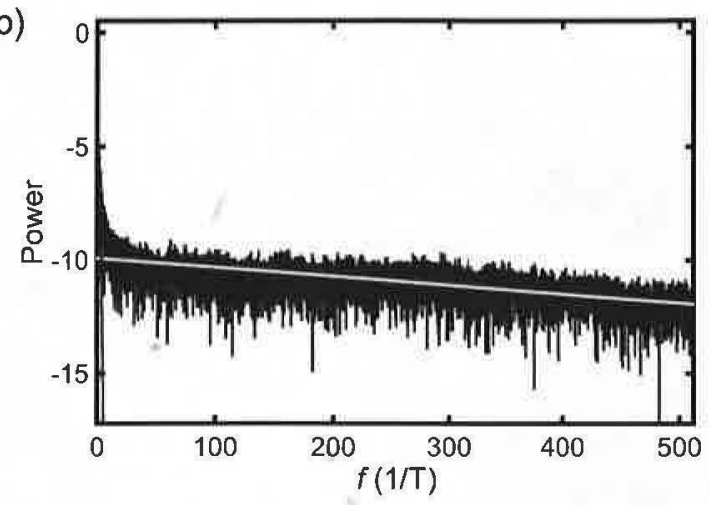

(c)

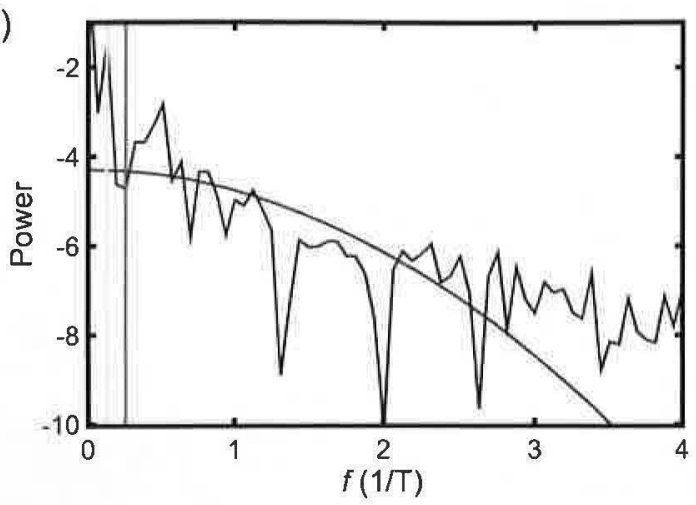

FIG. 11. Representative illustration of the FFT and Wiener analysis of MC characteristics of Pd atomic contacts. (a) The graph shows the original MC curve (blue), the low-frequency background (yellow), and the remaining fluctuation pattem (orange) after applying a band pass, which reduces the influence of the noise and removes low-frequency contributions. (b) The logarithmic power spectrum of the FFT of the original curve (blue). The Wiener noise filter is defined by a linear fit on the high-frequency contributions of the data (cyan). The essential signal range is determined by a polynomial fit (violet). (c) Shows a zoom-in of (b); the cutoff frequency for the long-wave contributions is indicated by the red line at $f_{c}=0.25 \mathrm{~T}^{-1}$. Right before this cutoff minimum, the spectra always show a pronounced peak (green line) corresponding to the dominant long-wave contributions.

$\delta G_{\text {ms. B.antisym. }}=0.0008 e^{2} / h$ is below the resolution limit of the measurement, while the amplitude of the symmetric part equals $\delta G_{\mathrm{rms} . \mathrm{B}}$.

\section{Fluctuation frequency analysis}

In a recent report on $\mathrm{Bi}$ atomic contacts, it has been shown that the long-wave $\mathrm{MC}$, originating from other effects, must be subtracted for the accurate interpretation of CFs [76]. This can be achieved by a fast Fourier transform (FFT) analysis where the long-wavelength contributions are subtracted using a high-pass filter with a certain frequency cutoff. Additionally, noise reduction is provided by a Wiener filter [77]. A typical example of the evaluation routine is shown in Fig. 11 for a single-atom Pd contact. Figure 11(a) shows the recorded MC trace as well as the filtered signal and the subtracted long-wave background. Figures 11(b) and 11(c) show the power spectrum of the FFT of the recorded data, as well as the corresponding low- and high-frequency fits of the Wiener analysis, where (c) is a zoom in the low-frequency region of (b). For all Pd and $\mathrm{Au}$ contacts we observe a local maximum in the frequency range of $0.1-0.2 \mathrm{~T}^{-1}$ of the power spectrum, which corresponds to the long-wave component of the MC. Therefore, the cutoff frequency $f_{\mathrm{c}}$ of the FFT low-pass filter is chosen at the first minimum after this peak in the power spectrum. Typically the cutoff frequencies, indicated by the red, vertical marker in Fig. 11(c), are narrowly distributed around $f_{\mathrm{c}}(\mathrm{Pd})=0.22 \mathrm{~T}^{-1}$ for Pd and $f_{c}(\mathrm{Au})=0.28 \mathrm{~T}^{-1}$ for $\mathrm{Au}$.

After subtraction of the long-wave components, the CF analysis of $\mathrm{Pd}$ contacts yields a fluctuation amplitude of $\delta \Delta G_{\mathrm{mms} . \mathrm{B}}=0.064 e^{2} / h$, which is of the same order of magnitude as $\delta G_{\text {ms. } v}$ but still more than twice the theoretically expected value. In contrast, for Au the FFT analysis yields a fluctuation amplitude of $\delta \Delta G_{\mathrm{ms} . \mathrm{B}}=1.4 \times 10^{-3} e^{2} / \mathrm{h}$, a value which is a factor of $\sim 7.8$ smaller than $\delta G_{\text {ms.v. }}$. Thus the FFT analysis reveals a different origin of the low-frequency contribution to the $\mathrm{MC}$ in $\mathrm{Au}$ and $\mathrm{Pd}$ atomic contacts. While for $\mathrm{Au}$ these long-wave components are in agreement with the expectations of CFs, for Pd their amplitude is larger than compatible with the theory, indicating an additional magnetotransport contribution.

Another important observation is the existence of a dominant cutoff frequency, which clearly separates the low- and high-frequency contributions in the MC curves. Since the characteristic length scales of $\mathrm{CF}$ are inversely proportional to the correlation field of the fluctuations, this confirms the contribution of two distinctly different scattering lengths. One 
of them corresponds to the inner part of the contact (long wave), while the second one is in the range of approximately hundred nanometer (short wave), which is the size of the lithographically defined bridge.

\section{SUMMARY AND CONCLUSION}

Atomic-size contacts represent a test-bed to explore the interplay between transport phenomena at the meso- and nanoscale. Here we provide a comprehensive investigation of magnetoconductance and electron-spectroscopic effects in $\mathrm{Pd}$ atomic contacts. The combination of different experiments and theoretical analysis provides strong evidence for the formation of local magnetic order in $\mathrm{Pd}$ in atomic-sized structures. The main arguments supporting this finding are: $\mathrm{Pd}$ shows a magnetoconductance behavior, which is comparable to atomic contacts of ferromagnetic transition metals as well as atomic chains of $\mathrm{Pt}$, where the emergence of magnetic order was recently confirmed. Secondly, the $d I / d V$ transport characteristics of a large fraction of Pd contacts reveals a zerobias anomaly that can be attributed to a Kondo resonance. The analysis shows that the occupation of the localized $d$ orbital in Pd is very similar to the one found in atomic contacts of the isoelectronic ferromagnet Ni. A fraction of Pd contacts did not show a Kondo resonance in the $d I / d V$ characteristics but an oscillatory behavior that can be interpreted as conduction fluctuations. However, the analysis of the corresponding MC spectra in terms of conductance fluctuations fails because the field-dependent fluctuation amplitude is too high, indicating the presence of scattering at a magnetic impurity. We believe that both local magnetic order and locally confined conductance fluctuations are a general phenomenon occurring at the atomic scale and are worth exploring in other elements as well.

\section{ACKNOWLEDGMENTS}

We are indebted to W. Belzig, U. Nowak, J. C. Cuevas, F. Pauly, C. Sürgers, and C. Untiedt for fruitful discussion and continuous cooperation. We gratefully acknowledge financial support from the Deutsche Forschungsgemeinschaft.
[1] N. Agraït, A. Levy Yeyati, and J. M. van Ruitenbeek, Quantum properties of atomic-sized conductors, Phys. Rep. 377, 81 (2003).

[2] J. K. Viljas, J. C. Cuevas, F. Pauly, and M. Häfner, Electronvibration interaction in transport through atomic gold wires, Phys. Rev. B 72, 245415 (2005).

[3] Y. G. Naidyuk and I. K. Yanson, Point-Contact Spectroscopy, Springer Series in Solid-State Sciences Vol. 145 (Springer, New York, 2004).

[4] I. K. Yanson, V. V. Fisun, R. Hesper, A. V. Khotkevich, J. M. Krans, J. A. Mydosh, and J. M. van Ruitenbeek, Size Dependence of Kondo Scattering in Point Contacts, Phys. Rev. Lett. 74, 302 (1995).

[5] B. Ludoph, M. H. Devoret, D. Esteve, C. Urbina, and J. M. van Ruitenbeek, Evidence for Saturation of Channel Transmission from Conductance Fluctuations in Atomic-Size Point Contacts, Phys. Rev. Lett. 82, 1530 (1999).

[6] B. V. Reddy, S. N. Khanna, and B. I. Dunlap, Giant Magnetic Moments in $4 d$ Clusters, Phys. Rev. Lett. 70, 3323 (1993).

[7] T. Taniyama, E. Ohta, and T. Sato, Observation of $4 d$ ferromagnetism in free-standing Pd fine particles, Europhys. Lett. 38, 195 (1997).

[8] T. Shinohara, T. Sato, and T. Taniyama, Surface Ferromagnetism of Pd Fine Particles, Phys. Rev. Lett. 91, 197201 (2003).

[9] B. Sampedro, P. Crespo, A. Hernando, R. Litrán, J. C. Sánchez López, C. López Cartes, A. Fernandez, J. Ramírez, J. González Calbet, and M. Vallet, Ferromagnetism in fcc Twinned $2.4 \mathrm{~nm}$ Size Pd Nanoparticles, Phys. Rev. Lett. 91, 237203 (2003).

[10] F. Aguilera-Granja, J. Montejano-Carrizales, and A. Vega, Magnetism in small Pd clusters, Phys. Lett. A 332, 107 (2004).

[11] X. Liu, M. Bauer, H. Bertagnolli, E. Roduner, J. van Slageren, and F. Phillipp, Structure and Magnetization of Small Monodisperse Platinum Clusters, Phys. Rev. Lett. 97, 253401 (2006).
[12] L. Fernández-Seivane and J. Ferrer, Magnetic Anisotropies of Late Transition Metal Atomic Clusters, Phys. Rev. Lett. 99, 183401 (2007).

[13] A. Delin, E. Tosatti, and R. Weht, Magnetism in Atomic-Size Palladium Contacts and Nanowires Phys. Rev. Lett. 92, 057201 (2004).

[14] Y. Mokrousov, G. Bihlmayer, S. Heinze, and S. Blügel, Giant Magnetocrystalline Anisotropies of $4 d$ Transition-Metal Monowires, Phys. Rev. Lett. 96, 147201 (2006).

[15] K. M. Smelova, D. I. Bazhanov, V. S. Stepanyuk, W. Hergert, A. M. Saletsky, and P. Bruno, Interplay between magnetism and structure in atomic-size Pd contacts: $A b$ initio studies, Phys. Rev. B 77, 033408 (2008).

[16] F. Pauly, M. Dreher, J. K. Viljas, M. Häfner, J. C. Cuevas, and P. Nielaba, Theoretical analysis of the conductance histograms and structural properties of $\mathrm{Ag}, \mathrm{Pt}$, and Ni nanocontacts, Phys. Rev. B 74, 235106 (2006).

[17] T. Nautiyal, T. H. Rho, and K. S. Kim, Nanowires for spintronics: A study of transition-metal elements of groups 8-10, Phys. Rev. B 69, 193404 (2004).

[18] P. Gava, A. Dal Corso, A. Smogunov, and E. Tosatti, Magnetisminduced ballistic conductance changes in palladium nanocontacts, Eur. Phys. J. B 75, 57 (2010).

[19] K. Ienaga, N. Nakashima, Y. Inagaki, H. Tsujii, T. Kimura, and T. Kawae, Study of ferromagnetic transition in Pd nanometer-scale constrictions using a mechanically controllable break junction technique, Appl. Phys. Lett. 101, 123114 (2012).

[20] G. D. Scott, J. J. Palacios, and D. Natelson, Anomalous transport and possible phase transition in palladium nanojunctions, ACS Nano 4, 2831 (2010).

[21] F. Strigl, C. Espy, M. Bückle, E. Scheer, and T. Pietsch, Emerging magnetic order in platinum atomic contacts and chains, Nat. Commun. 6, 6172 (2015). 
[22] P. Błoński and J. Hafner, Magneto-structural properties and magnetic anisotropy of small transition-metal clusters: A firstprinciples study, J. Phys.: Condens. Matter 23, 136001 (2011).

[23] M. J. Piotrowski, P. Piquini, and J. L. F. Da Silva, Density functional theory investigation of $3 d, 4 d$, and $5 d 13$-atom metal clusters, Phys. Rev. B 81, 155446 (2010).

[24] N. Watari and S. Ohnishi, Atomic and electronic structures of $\mathrm{Pd}_{13}$ and $\mathrm{Pt}_{13}$ clusters, Phys. Rev. B 58, 1665 (1998).

[25] G. Ganteför and W. Eberhardt, Localization of $3 d$ and $4 d$ Electrons in Small Clusters: The "Roots" of Magnetism, Phys. Rev. Lett. 76, 4975 (1996).

[26] M. Reyes Calvo, J. Fernández-Rossier, J. J. Palacios, D. Jacob, D. Natelson, and C. Untiedt, The Kondo effect in ferromagnetic atomic contacts, Nature (London) 458, 1150 (2009).

[27] M. R. Calvo, D. Jacob, and C. Untiedt, Analysis of the Kondo effect in ferromagnetic atomic-sized contacts, Phys. Rev. B 86, 075447 (2012).

[28] J. M. van Ruitenbeek, A. Alvarez, I. Piñeyro, C. Grahmann, P. Joyez, M. H. Devoret, D. Esteve, and C. Urbina, Adjustable nanofabricated atomic size contacts, Rev. Sci. Instrum. 67, 108 (1996).

[29] S. Csonka, A. Halbritter, G. Mihály, O. I. Shklyarevskii, S. Speller, and H. van Kempen, Conductance of Pd-H Nanojunctions, Phys. Rev. Lett. 93, 016802 (2004).

[30] P. Kumar, R. Skomski, P. Manchanda, and A. Kashyap, Ab-initio study of anisotropy and nonuniaxial anisotropy coefficients in Pd nanochains, Chem. Phys. Lett. 583, 109 (2013).

[31] B. Zhang, D. Wang, Z. Yang, L. Xu, R. Liu, P. Li, and X. Li, Non-collinear magnetic order and spin-orbit coupling effect in $4 d$ transition metal monatomic chains, J. Magn. Magn. Mater. 368, 262 (2014).

[32] N. García, M. Muñoz, and Y.-W. Zhao, Magnetoresistance in Excess of $200 \%$ in Ballistic Ni Nanocontacts at Room Temperature and 100 Oe, Phys. Rev. Lett. 82, 2923 (1999).

[33] M. R. Sullivan, D. A. Boehm, D. A. Ateya, S. Z. Hua, and H. D. Chopra, Ballistic magnetoresistance in nickel single-atom conductors without magnetostriction, Phys. Rev. B 71, 024412 (2005).

[34] Z. K. Keane, L. H. Yu, and D. Natelson, Magnetoresistance of atomic-scale electromigrated nickel nanocontacts, Appl. Phys. Lett. 88, 062514 (2006).

[35] S.-F. Shi and D. C. Ralph, Atomic motion in ferromagnetic break junctions, Nat. Nanotechnol. 2, 522 (2007).

[36] S. Egle, C. Bacca, H.-F. Pernau, M. Huefner, D. Hinzke, U. Nowak, and E. Scheer, Magnetoresistance of atomic-size contacts realized with mechanically controllable break junctions, Phys. Rev. B 81, 134402 (2010).

[37] M. Müller, R. Montbrun, M. Marz, V. Fritsch, C. Sürgers, and H. v. Löhneysen, Switching the conductance of Dy nanocontacts by magnetostriction, Nano Lett. 11, 574 (2011).

[38] W. F. Egelhoff, Jr., L. Gan, H. Ettedgui, Y. Kadmon, C. J. Powell, P. J. Chen, A. J. Shapiro, R. D. McMichael, J. J. Mallett, T. P. Moffat, M. D. Stiles, and E. B. Svedberg, Artifacts that mimic ballistic magnetoresistance, J. Magn. Magn. Mater. 287, 496 (2005).

[39] M. Häfner, J. K. Viljas, and J. C. Cuevas, Theory of anisotropic magnetoresistance in atomic-sized ferromagnetic metal contacts, Phys. Rev. B 79, 140410 (2009).

[40] K. I. Bolotin, F. Kuemmeth, and D. C. Ralph, Anisotropic Mag netoresistance and Anisotropic Tunneling Magnetoresistance
Due to Quantum Interference in Ferromagnetic Metal Break Junctions, Phys. Rev. Lett. 97, 127202 (2006).

[41] M. Viret, M. Gabureac, F. Ott, C. Fermon, C. Barreteau, G. Autes, and R. Guirado-Lopez, Giant anisotropic magnetoresistance in ferromagnetic atomic contacts, Eur. Phys. J. B 51, 1 (2006).

[42] E. Scheer, N. Agraït, J. C. Cuevas, A. Levy Yeyati, B. Ludoph, A. Martín-Rodero, G. Rubio Bollinger, J. M. van Ruitenbeek, and C. Urbina, The signature of chemical valence in the electrical conduction through a single-atom contact, Nature (London) 394, 154 (1998)

[43] J. C. Cuevas, A. Levy Yeyati, A. Martín-Rodero, G. Rubio Bollinger, C. Untiedt, and N. Agrait, Evolution of Conducting Channels in Metallic Atomic Contacts under Elastic Deformation, Phys. Rev. Lett. 81, 2990 (1998).

[44] J. Heurich, J. C. Cuevas, W. Wenzel, and G. Schön, Electrical Transport through Single-Molecule Junctions: From Molecular Orbitals to Conduction Channels, Phys. Rev. Lett. 88, 256803 (2002).

[45] R. H. M. Smit, C. Untiedt, A. I. Yanson, and J. M. van Ruitenbeek, Common Origin for Surface Reconstruction and the Formation of Chains of Metal Atoms, Phys. Rev. Lett. 87, 266102 (2001).

[46] A. Thiess, Y. Mokrousov, S. Heinze, and S. Blügel, Magnetically Hindered Chain Formation in Transition-Metal Break Junctions, Phys. Rev. Lett. 103, 217201 (2009).

[47] L. Xiao and L. Wang, Structures of platinum clusters: Planar or spherical? J. Phys. Chem. A 108, 8605 (2004).

[48] W. Zhang, Q. Ge, and L. Wang, Structure effects on the energetic, electronic, and magnetic properties of palladium nanoparticles, J. Chem. Phys. 118, 5793 (2003).

[49] J. Wang, G. Wang, and J. Zhao, Density-functional study of $\mathrm{Au}_{\mathrm{n}}$ ( $n=2-20$ ) clusters: Lowest-energy structures and electronic properties, Phys. Rev. B 66, 035418 (2002).

[50] J. A. Alonso, Electronic and atomic structure, and magnetism of transition-metal clusters, Chem. Rev. (Washington, DC, U. S.) 100, 637 (2000).

[51] J. C. Tung and G. Y. Guo, Magnetic moment and magnetic anisotropy of linear and zigzag $4 d$ and $5 d$ transition metal nanowires: First-principles calculations, Phys. Rev. B 81, 094422 (2010).

[52] A. Smogunov, A. Dal Corso, and E. Tosatti, Magnetic phenomena, spin-orbit effects, and Landauer conductance in Pt nanowire contacts: Density-functional theory calculations, Phys. Rev. B 78, 014423 (2008).

[53] A. Thiess, Y. Mokrousov, and S. Heinze, Competing magnetic anisotropies in atomic-scale junctions, Phys. Rev. B 81, 054433 (2010).

[54] J. Kondo, Effect of ordinary scattering on exchange scattering from magnetic impurity in metals, Phys. Rev. 169, 437 (1968).

[55] P. W. Anderson, Localized magnetic states in metals, Phys. Rev. 124, 41 (1961).

[56] M. Ternes, Spin excitations and correlations in scanning tunneling spectroscopy, New J. Phys. 17, 063016 (2015).

[57] T. Uchihashi, J. Zhang, J. Kröger, and R. Berndt, Quantum modulation of the Kondo resonance of $\mathrm{Co}$ adatoms on $\mathrm{Cu} / \mathrm{Co} / \mathrm{Cu}(100)$ : Low-temperature scanning tunneling spectroscopy study, Phys. Rev. B 78, 033402 (2008). 
[58] S. M. Cronenwett, T. H. Oosterkamp, and L. P. Kouwenhoven, A tunable Kondo effect in quantum dots, Science 281, 540 (1998).

[59] A. C. Hewson, The Kondo Problem to Heavy Fermions (Cambridge University Press, Cambridge, UK, 1997), Vol. 2.

[60] C. P. Umbach, S. Washburn, R. B. Laibowitz, and R. A. Webb, Magnetoresistance of small, quasi-one-dimensional, normalmetal rings and lines, Phys. Rev. B 30, 4048 (1984).

[61] P. A. Lee and A. D. Stone, Universal Conductance Fluctuations in Metals, Phys. Rev. Lett. 55, 1622 (1985).

[62] P. A. Lee, A. D. Stone, and H. Fukuyama, Universal conductance fluctuations in metals: Effects of finite temperature, interactions, and magnetic field, Phys. Rev. B 35, 1039 (1987).

[63] B. L. Al'tshuler and D. E. Khmel'nitskii, Fluctuation properties of small conductors, Pis'ma Zh. Eksp. Teor. Fiz. 42, 291 (1985) [Soviet J. Exp. Theor. Phys. Lett. 42, 359 (1985)].

[64] D. E. Khmel'nitskii and A. I. Larkin, Nonlinear conductance in the mesoscopic regime, Phys. Scr. T14, 4 (1986).

[65] P. A. M. Holweg, J. A. Kokkedee, J. Caro, A. H. Verbruggen, S. Radelaar, A. G. M. Jansen, and P. Wyder, Conductance Fluctuations in a Ballistic Metallic Point Contact, Phys. Rev. Lett. 67, 2549 (1991),

[66] B. Ludoph and J. M. van Ruitenbeek, Conductance fluctuations as a tool for investigating the quantum modes in atomic-size metallic contacts, Phys. Rev. B 61, 2273 (2000).

[67] J. C. Licini, D. J. Bishop, M. A. Kastner, and J. Melngailis, Aperiodic Magnetoresistance Oscillations in Narrow Inversion Layers in Si, Phys. Rịev. Lett. 55, 2987 (1985).

[68] J. R. Gao, J. Caro, A. H. Verbruggen, S. Radelaar, and J. Middelhoek, Temperature dependence of universal conductance fluctuations in narrow mesoscopic Si inversion layers, Phys. Rev. B 40, 11676 (1989).

[69] P. A. M. Holweg, J. Caro, A. H. Verbruggen, and S. Radelaar, Correlation energy of conductance fluctuations in ballistic silver point contacts, Phys. Rev. B 48, 2479 (1993).

[70] D. C. Ralph, K. S. Ralls, and R. A. Buhrman, Ensemble Studies of Nonlinear Conductance Fluctuations in Phase Coherent Samples, Phys. Rev. Lett. 70, 986 (1993).

[71] P. H. Dederichs, H. Schober, and D. J. Sellmyer, LandoltBörnstein - Group III Condensed Matter 13 a (Phonon States of Elements. Electron States and Fermi Surfaces of Alloys) (Springer, Berlin, Heidelberg, 1981).

[72] N. Agrait, C. Untiedt, G. Rubio-Bollinger, and S. Vieira, Onset of Energy Dissipation in Ballistic Atomic Wires, Phys. Rev. Lett. 88, 216803 (2002).

[73] T. Böhler, A. Edtbauer, and E. Scheer, Point-contact spectroscopy on aluminium atomic-size contacts: Longitudinal and transverse vibronic excitations, New J. Phys. 11, 013036 (2009).

[74] R. Landauer, Electrical resistance of disordered onedimensional lattices, Philos. Mag. 21, 863 (1970).

[75] Y. V. Sharvin, A possible method for studying Fermi surfaces, Zh. Eksp. Teor. Fiz. 48, 984 (1965) [Sov. Phys. JETP 21, 655 (1965)].

[76] H.-F. Pernau, T. Pietsch, and E. Scheer, Magnetotransport in atomic-size bismuth contacts, J. Phys.: Condens. Matter 26, 474203 (2014).

[77] W. H. Press, S. A. Teukolsky, W. T. Vetterling, and B. P. Flannery, Numerical Recipes: The Ant of Scientific Computing, 3rd ed. (Cambridge University Press, New York, 2007). 